\title{
Multiple Resonances in the Semi-Classical Limit
}

\author{
Noureddine Kaidi ${ }^{1}$ and Michel Rouleux ${ }^{2}$ \\ 1 Faculté des Sciences de Tunis, Département de Mathématiques, 1060 Tunis, Tunisia \\ 2 CNRS Luminy, Case 907, CPT, F-13288 Marseille Cedex, France and \\ Université de Toulon et du Var, PHYMAT, F-83130 La Garde, France
}

Received February 6, 1990

\begin{abstract}
We construct for the Schrödinger operator in the semi-classical limit compact perturbations of a radial symmetric potential which give rise to resonances associated to arbitrarily high order poles for the meromorphic extension of the resolvent. Our results concern the hamiltonian $P_{0}=-h^{2} \Delta-x^{2}$ in the 2-dimensional case, as well as a fairly large class of radial-symmetric potentials in the 3-dimensional case. We show that the poles of the resolvent for such a potential are necessarily simple, and subsequently the degeneracy is due to a lack of symmetry.
\end{abstract}

\section{Introduction}

We consider the Schrödinger operator in semi-classical limit on $L^{2}\left(\mathbb{R}^{n}\right)$ :

$$
P=-h^{2} \Delta+V(x) \quad(h \rightarrow 0) .
$$

If $V \in \mathscr{C}^{\infty}\left(\mathbb{R}^{n}: \mathbb{R}\right)$ satisfies:

$$
\lim _{|x| \rightarrow \infty} V(x)=E_{0}>-\infty,
$$

then $P$ is essentially self-adjoint on $\mathscr{C}_{0}^{\infty}\left(\mathbb{R}^{n}\right)$ as an unbounded operator, and $L^{2}\left(\mathbb{R}^{n}\right)=\mathscr{H}_{\mathrm{pp}} \oplus \mathscr{H}_{\mathrm{ess}}$, where $\mathscr{H}_{\mathrm{pp}}$ is the sum of the bound state corresponding to the pure point spectrum $\sigma_{\mathrm{pp}}(P)$ and $\mathscr{H}_{\text {ess }}$ the space of free states associated to the essential spectrum $\sigma_{\text {ess }}(P)$. Here the essential spectrum coincides with the absolutely continuous spectrum. The threshold $E_{0}$ is the bottom of the essential spectrum

$$
\inf \sigma_{\text {ess }}(P)=E_{0} .
$$

In particular, if $E_{0}>0$, there is only discrete spectrum near 0 and for $z \in \mathbb{C}$ small enough, the resolvent $R(z)=(P-z)^{-1}$ is a meromorphic function whose poles (necessarily simple) are the (real) eigenvalues of $P$. 
If $E_{0}<0, R(z)$ is an holomorphic function in the half lower plane $\operatorname{Im} z<0$ and half upper plane $\operatorname{Im} z>0$ for $z$ near 0 . Under some additional conditions, $R(z)$ extends across the real axis in the other half plane as a meromorphic function whose poles are called resonances. In the classical theories of Aguilar-Combes [AgCo], Balslev-Combes [BaCo], and Helffer-Sjostrand [HeSj], the extension of $R(z)$ appears as the resolvent of a non-self adjoint operator whose poles are not to be simple. Ramm [Ra] has studied stability of the algebraic multiplicity $q$ of a resonance for the Schrödinger operator. Sjöstrand [Sj], in the semi-classical frame, proved existence of a double pole for some perturbation of $P_{0}(h)=-h^{2} \Delta-x^{2}$ in $\mathbb{R}^{2}$, starting from a resonance with geometric multiplicity equal to 2 . Here we extend this property by showing, under some suitable hypotheses, that any resonance $\lambda(h)$ with multiplicity $q$ (simple pole of the resolvent) of a radial symmetric hamiltonian $P=-h^{2} \Delta+V$, can give rise, under some slight, compact perturbation $\Delta V$ of the potential, to a pole of order $q$, or in other terms, that the matrix of $\widetilde{P}=P+\Delta V$ relative to the invariant subspace derived from $\operatorname{Ker}(P-\lambda(h))$ has a nilpotent part of order $q$. Let us point out that this result is relevant to the well-known fact there is no general a priori estimate for the norm of the resolvent $(P-z)^{-1}$ in terms of some fixed power of the distance of $z$ to the spectrum of $P$, when $P$ is not self-adjoint.

Our results apply to the hamiltonian $P_{0}=-h^{2} \Delta-|x|^{2}$ in two dimensions, as well as to a fairly general class of spherical symmetric potentials in three dimensions. By the way, we show that the poles of such a potential are necessarily simple, and the degeneracy is due subsequently to a breaking of symmetry. We will see also how the multiplicity of the resonances is generally bounded by the number of spherical harmonics associated to a given orbital momentum; in that sense the case of the hamiltonian $P_{0}$ is exceptional, particular to the 2-dimensional case, which nevertheless allows us to give relatively simple examples where multiple resonances occur. We finally give in the Appendix a semi-classical result of classification of levels $E_{n, l}$ for the resonant states in 3 dimensions, which can be carried out, mutatis mutandis, in the self-adjoint case.

The results of this paper have been announced in [KaRo].

The authors want to thank J. Sjöstrand for motivating discussions.

\section{Perturbation Matrix}

We extend a little in this section the constructions of [Sj], adapting them in ordinary complex scaling in order to simplify some of the proofs. For equivalence of the various definitions we refer to Helffer and Martinez [HeMa].

Let $P=-h^{2} \Delta+V$ the Schrödinger operator on $L^{2}\left(\mathbb{R}^{n}\right)$ with $V \in \mathscr{C}^{\infty}\left(\mathbb{R}^{n} ; \mathbb{R}\right)$ satisfying:

$V$ is analytic on $\mathbb{R}^{n}$ and dilation analytic, i.e. $V$ has an analytic extension in:

$$
G=\{x \in \mathbb{C}:|\operatorname{Im} x| \leqq C\langle\operatorname{Re} x\rangle\}, \quad \text { where }\langle x\rangle=\left(1+x^{2}\right)^{1 / 2} .
$$

$V$ verifies the virial condition outside $x=0$ at energy 0 , i.e. for any $\delta>0$ there exists $C>0$ such that:

$$
\begin{gathered}
-2 V(x)-x \cdot \nabla V(x) \geqq C \text { for } \quad x \in \mathbb{R}^{n}, \quad|x|>\delta . \\
V(0)=0 \text { and } V \text { has a non-degenerate maximum at } x=0 . \\
\lim _{|x| \rightarrow \infty, x \in G} V(x)=E_{0}<0 .
\end{gathered}
$$


Hypothesis (1.2) is satisfied in particular if:

$V$ is radial symmetric $\left(V(x)=V\left(|x|^{2}\right)\right)$ and $V(x)<0, \partial_{|x|} V<0$ for $x \neq 0$.

Let $p(x, \xi)=\xi^{2}+V(x)$ the classical hamiltonian associated to $P$, and $K \subset p^{-1}(0) \cap \mathbb{R}^{2 n}$ the set of trapped trajectories for $p$, i.e.

$$
\forall \varrho \in K: \exp t H_{p}(\varrho) \rightarrow \infty \text { as } t \rightarrow \pm \infty \text {. }
$$

Then assumptions (1.1) to (1.3) ensure $K=\{(0,0)\}$. Let $U_{\theta}$ be the one parameter family of dilations defined by: $U_{\theta} \varphi(x)=e^{\frac{n \theta}{2}} \varphi\left(e^{\theta} x\right)$. For real $\theta, U_{\theta}$ is unitary on $L^{2}\left(\mathbb{R}^{n}\right)$. Let:

$$
P_{\theta}=U_{\theta} P U_{\theta}^{-1}=-h^{2} e^{-2 \theta} \Delta+V\left(e^{\theta} x\right) .
$$

Under hypotheses (1.1) to (1.4) the $P_{\theta}$ extend to an analytic family of type $(A)$ of operators on $L^{2}\left(\mathbb{R}^{n}\right)$ for $|\operatorname{Im} \theta|$ small enough. For $\operatorname{Im} \theta \neq 0, P_{\theta}$ has discrete spectrum near 0 , whose eigenvalues are the resonances of $P$. Resonances for $\operatorname{Im} \theta>0$ are called "physical" or "outgoing," others are called "incoming" resonances. In the complex scaling theory, it is useful to consider the scalar product on $L^{2}\left(\mathbb{R}^{n}\right)$ as a duality product between $L^{2}\left(\Gamma_{\theta}\right)=L^{2}\left(e^{\theta} \mathbb{R}^{n}\right)$ and $L^{2}\left(\Gamma_{\bar{\theta}}\right)=L^{2}\left(e^{\theta} \mathbb{R}^{n}\right)$ by means of the formula:

$$
(u \mid v)=\int_{e^{\theta \mathbb{R}^{n}}} u(y) \overline{v(\bar{y})} d y .
$$

We will identify $L^{2}\left(\Gamma_{\theta}\right)$ and $L^{2}\left(\Gamma_{\bar{\theta}}\right)$ to $L^{2}\left(\mathbb{R}^{n}\right) \equiv L^{2}$.

We also consider the hamiltonian $P_{0}=-h^{2} \Delta-|x|^{2}$. The resonant functions of $P_{0}$ associated to the outgoing resonances:

$$
\lambda_{0}(h)=-i h(2 \sigma+n) \quad(\sigma \in \mathbb{N})
$$

are spanned by the Hermite functions:

$$
\varphi_{\alpha}=C_{\alpha} h^{-\frac{(2 \sigma+n)}{4}} \tilde{\Phi}_{\alpha}\left(h^{-1 / 2} x\right) e^{i x^{2} / 2 h},
$$

where $\alpha \in \mathbb{N}^{n},|\alpha|=\sigma$, and $\widetilde{\Phi}_{\alpha}$ is an Hermite polynomial. The incoming resonances of $P_{0}$ are then:

$$
\mu_{0}(h)=i h(2 \sigma+n)
$$

and the associated resonant functions:

$$
\varphi_{\alpha}^{*}=\text { holomorphic extension of } \bar{\varphi}_{\alpha}(x), \quad x \in \mathbb{R}^{n} .
$$

The constant $C_{\alpha} \in \mathbb{C}$ is determined by the normalisation condition $\left(\varphi_{\alpha} \mid \varphi_{\alpha}^{*}\right)=1$ for the duality between $L^{2}\left(\Gamma_{\theta}\right)$ and $L^{2}\left(\Gamma_{\bar{\theta}}\right)$. Here we can analytically extend up to $\operatorname{Im} \theta=\frac{\pi}{4}$. We know then $[\mathrm{Sj}, \mathrm{BrCoDu}]$ that for any $C_{0}>0$ such that none of the values (1.5) belongs to the boundary of $D\left(0, C_{0} h\right)$, and for any $h>0$ small enough, there are bijections $b^{ \pm}=b^{ \pm}(h)$ from the set of resonances of $P_{0}$ :

$$
\Gamma_{0}^{ \pm}(h)=\{\mp i h(2 \sigma+n) \mid \sigma \in \mathbb{N}\} \cap D\left(0, C_{0} h\right)
$$


onto the set $\Gamma^{ \pm}(h)$ of resonances of $P$ (counted with their multiplicity) inside $D\left(0, C_{0} h\right)$ such that:

$$
b^{ \pm}(E)-E=\mathcal{O}\left(h^{3 / 2}\right) \quad(h \rightarrow 0) .
$$

[Under the radial symmetry assumption (1.2) we actually get $\mathcal{O}\left(h^{2}\right)$ ]. If $F_{\lambda} \subset L^{2}\left(\Gamma_{\theta}\right)$ for $\operatorname{Im} \theta>0$ [respectively $\left.F_{\mu}^{\prime} \subset L^{2}\left(\Gamma_{\bar{\theta}}\right)\right]$ is the space of resonant functions associated to $\lambda(h) \in \Gamma^{+}(h)\left[\right.$ respectively $\left.\mu(h) \in \Gamma^{-}(h)\right]$, then $F_{\lambda} \perp F_{\mu}^{\prime}$ if $\lambda(h) \neq \bar{\mu}(h)$ and $F_{\lambda}=\left(F_{\mu}^{\prime}\right) *$ if $\lambda(h)=\bar{\mu}(h)$. For $\varepsilon_{0}>0$ small enough, and $D=D\left(\lambda_{0}(h), \varepsilon_{0} h\right)$, we know that:

$$
\forall z \in \partial D:\left(P_{\theta}-z\right)^{-1}=\mathcal{O}\left(h^{-1}\right): L^{2} \rightarrow L^{2}
$$

uniformly for $h>0$ small enough. Let

$$
\Pi=\frac{1}{2 i \pi} \int_{\partial D}\left(z-P_{\theta}\right)^{-1} d z: L^{2} \rightarrow L^{2}
$$

the spectral projector of $P_{\theta}$ in $D$, and $G=\Pi\left(L^{2}\right)$. For $\varepsilon_{0}>0$ small enough, the rank of $\Pi$ is equal to the multiplicity $q_{0}=\left(\begin{array}{c}n+\sigma-1 \\ \sigma\end{array}\right)$ of $\lambda_{0}(h)$. We then make the following assumption on $\lambda(h) \in \Gamma^{+}(h)$ :

$$
\exists \delta>0, \exists N \geqq 1 \quad \text { such that } D\left(\lambda(h), 2 \delta h^{N}\right) \cap \Gamma^{+}(h)=\{\lambda(h)\} .
$$

Remark 1.1. Hypothesis (1.9) is generic: for odd $n, V(x)=-|x|^{2}+\alpha|x|^{4}+\mathcal{O}\left(|x|^{6}\right)$ $(x \rightarrow 0)$ with $\alpha \neq 0$, we will show in the Appendix that (1.9) is satisfied for $N=2$.

We get the following:

Lemma 1.2. $\exists N_{1} \geqq 1$ such that $\forall z \in \partial D\left(\lambda(h), \partial h^{N}\right):\left(P_{\theta}-z\right)^{-1}=\mathcal{O}\left(h^{-N_{1}}\right): L^{2} \rightarrow L^{2}$ uniformly for $h>0$ small enough.

Proof. We have:

$$
\left(P_{\theta}-z\right)^{-1}=\left(P_{\theta}-z\right)^{-1} \Pi+\left(P_{\theta}-z\right)^{-1}(I-\Pi) .
$$

Let $\tilde{D}=D\left(\lambda(h), \delta h^{N}\right)$. For $z \in \partial \tilde{D}$, and $h>0$ small enough, the first resolvent formula shows:

$$
\left(P_{\theta}-z\right)^{-1}(I-\Pi)=\frac{1}{2 i \pi} \int_{\partial D}(\zeta-z)^{-1}\left(P_{\theta}-\zeta\right)^{-1} d \zeta .
$$

Formula (1.8) and estimate: $\forall z \in \partial \tilde{D}, \forall \zeta \in \partial D: C^{-1} h \leqq|z-\zeta| \leqq C h(C>1)$ show

$$
\forall z \in \partial \tilde{D}:\left(P_{\theta}-z\right)^{-1}(I-\Pi)=\mathcal{O}\left(h^{-1}\right): L^{2} \rightarrow L^{2} .
$$

We then consider $\left(P_{\theta}-z\right)^{-1} \Pi=\Pi\left(P_{\theta}-z\right)^{-1} \Pi$. For $h>0$ small enough, the family of functions:

$$
w_{\alpha}=\Pi U_{\theta} \varphi_{\alpha}, \quad|\alpha|=\sigma
$$

are a basis of $G$. In the same way, we consider, for $z \in \partial D^{*}=\partial D\left(\mu_{0}(h), \varepsilon_{0} h\right)$ the resolvent $\left(P_{\bar{\theta}}-z\right)^{-1}: L^{2} \rightarrow L^{2}$ and the spectral projector

$$
\Pi^{*}=\frac{1}{2 i \pi} \int_{\partial D^{*}}\left(z-P_{\bar{\theta}}\right)^{-1} d z
$$

associated to the incoming resonances of $P$ in $D\left(\mu_{0}(h), \varepsilon_{0} h\right)$. The subspace $G^{*}=\Pi^{*}\left(L^{2}\right)$ is a sum of characteristic subspaces for $P_{\bar{\theta}}$, with dimension $q_{0}$, dual of 
the latter. Let $\left(w_{\beta}^{*}\right)_{|\beta|=\sigma}$ the dual basis of the $\left(w_{\alpha}\right)$. Then the matrix of $P_{\theta}\left\lceil G=\Pi P_{\theta} \Pi\right.$ is $M=\left(P_{\theta} w_{\alpha} \mid w_{\beta}^{*}\right)_{|\alpha|=|\beta|=\sigma}$. We have the following:

Lemma 1.3. Under the hypothesis (1.1) to (1.4) we have:

$$
\begin{gathered}
w_{\alpha}=U_{\theta} \varphi_{\alpha}+\mathcal{O}\left(h^{1 / 2}\right) \quad \text { in } L^{2}, \\
\left(P_{\theta} w_{\alpha} \mid w_{\beta}^{*}\right)=\lambda_{0}(h) \delta_{\alpha \beta}+\mathcal{O}\left(h^{3 / 2}\right),
\end{gathered}
$$

and under the spherical symmetry assumption $(1.2)^{\prime}$ we get respectively $\mathcal{O}(h)$ and $\mathcal{O}\left(h^{2}\right)$ in (1.12) and (1.13).

Proof. The second part of the lemma follows from a mere analysis of the proof. We use the geometrical resolvent estimates of $[\mathrm{BrCoDu}]$. Let $J_{i}, J_{e} \in \mathscr{C}^{\infty}\left(\mathbb{R}^{n}\right)$, suitably chosen with $J_{i}=1$ near $0, J_{e}=1$ near infinity, satisfying the condition $J_{i}^{2}+J_{e}^{2}=1$. We introduce the identification operator:

$$
J: L^{2}\left(\mathbb{R}^{n}\right) \oplus L^{2}\left(\operatorname{supp} J_{e}\right) \rightarrow L^{2}\left(\mathbb{R}^{n}\right) \quad J(u \oplus v)=J_{i} u+J_{e} v
$$

and its adjoint:

$$
J^{*}: L^{2}\left(\mathbb{R}^{n}\right) \rightarrow L^{2}\left(\mathbb{R}^{n}\right) \oplus L^{2}\left(\operatorname{supp} J_{e}\right) \quad J^{*}(u)=J_{i} u \oplus J_{e} u .
$$

We then define:

$$
P_{\theta}^{i}=-h^{2} e^{-2 \theta} \Delta+e^{2 \theta} V_{0}(x) \text { on } L^{2}\left(\mathbb{R}^{n}\right),
$$

where $V_{0}(x)=\frac{1}{2}\left\langle V^{\prime \prime}(0) x, x\right\rangle$, with domain $\mathscr{D}\left(P_{\theta}^{i}\right)=\mathscr{D}(\Delta) \cap \mathscr{D}\left(|x|^{2}\right)$ (quadratic approximation), $P_{\theta}^{e}$ as the Dirichlet realization of $P_{\theta}$ in $L^{2}\left(\operatorname{supp} J_{e}\right)$ and $P_{\theta}^{d}=P_{\theta}^{i} \oplus P_{\theta}^{e}$. We recall the resolvent equation

$$
\left(P_{\theta}-z\right)^{-1}=J\left(P_{\theta}^{d}-z\right)^{-1} J^{*}-\left(P_{\theta}-z\right)^{-1} \Pi_{0}\left(P_{\theta}^{d}-z\right)^{-1} J^{*},
$$

where $\Pi_{0}$ is the operator defined by

$$
\Pi_{0}(u \oplus v)=W J_{i} u-h^{2} e^{-2 \theta}\left(\left[\Delta, J_{i}\right] u+\left[\Delta, J_{e}\right] v\right)
$$

with

$$
W=V\left(e^{\theta} x\right)+e^{2 \theta} V_{0}(x)
$$

We get

$$
\left(P_{\theta}-z\right)^{-1} U_{\theta} \varphi_{\alpha}=\left(\lambda_{0}(h)-z\right)^{-1} U_{\theta} \varphi_{\alpha}+\left(\lambda_{0}(h)-z\right)^{-1}\left(P_{\theta}-z\right)^{-1} W U_{\theta} \varphi_{\alpha},
$$

and by integration along the boundary of $D$ :

$$
w_{\alpha}=U_{\theta} \varphi_{\alpha}+\frac{1}{2 i \pi} \int_{\partial D}\left(\lambda_{0}(h)-z\right)^{-1}\left(P_{\theta}-z\right)^{-1} W U_{\theta} \varphi_{\alpha} d z .
$$

Equation (1.14) gives:

$$
\begin{aligned}
\left(P_{\theta}-z\right)^{-1} W U_{\theta} \varphi_{\alpha}= & J_{i}\left(P_{\theta}^{i}-z\right)^{-1} J_{i} W U_{\theta} \varphi_{\alpha}+J_{e}\left(P_{\theta}-z\right)^{-1} J_{e} W U_{\theta} \varphi_{\alpha} \\
& -\left(P_{\theta}-z\right)^{-1} W J_{i}\left(P_{\theta}^{i}-z\right)^{-1} J_{i} W U_{\theta} \varphi_{\alpha} \\
& +h^{2} e^{-2 \theta}\left(P_{\theta}-z\right)^{-1}\left[\Delta, J_{i}\right]\left(P_{\theta}^{i}-z\right)^{-1} J_{i} W U_{\theta} \varphi_{\alpha} \\
& +h^{2} e^{-2 \theta}\left(P_{\theta}-z\right)^{-1}\left[\Delta, J_{e}\right]\left(P_{\theta}^{e}-z\right)^{-1} J_{e} W U_{\theta} \varphi_{\alpha} .
\end{aligned}
$$

We set $U \varphi(x)=h^{-n / 4} \varphi\left(h^{-1 / 2} x\right)$. $U$ is unitary on $L^{2}$ and:

$$
\left(P_{\theta}^{i}-z\right)^{-1} J_{i} W=h^{-1} U\left(Q_{\theta}^{i}-h^{-1} z\right)^{-1} J_{i}\left(h^{1 / 2} x\right) W\left(h^{1 / 2} x\right) U^{-1}
$$


with $Q_{\theta}^{i}=-e^{-2 \theta} \Delta+e^{2 \theta} V_{0}$. For $z \in \partial D$, we easily show [BrCoDu]: $\left(Q_{\theta}^{i}-h^{-1} z\right)^{-1}$ $=\mathcal{O}(1): L^{2} \rightarrow L^{2}$. There follows:

$$
\begin{aligned}
\left(P_{\theta}^{i}-z\right)^{-1} J_{i} W U_{\theta} \varphi_{\alpha} & =h^{-1} U\left(Q_{\theta}^{i}-h^{-1} z\right)^{-1} J_{i}\left(h^{1 / 2} x\right) W\left(h^{1 / 2} x\right)\langle x\rangle^{-3} \\
& \times\langle x\rangle^{3} U^{-1} U_{\theta} \varphi_{\alpha}=\mathcal{O}\left(h^{1 / 2}\right)
\end{aligned}
$$

for $W\left(h^{1 / 2} x\right)\langle x\rangle^{-3}=\mathcal{O}\left(h^{3 / 2}\right)$ in $L^{\infty}$ and $\langle x\rangle^{3} U^{-1} U_{\theta} \varphi_{\alpha}=\mathcal{O}(1)$. Let $h^{-1}\left(Q_{\theta}-h^{-1} z\right)^{-1}=U^{-1}\left(P_{\theta}-z\right)^{-1} U$. The estimate (1.8) shows:

$$
\left(Q_{\theta}-h^{-1} z\right)^{-1}=\mathcal{O}(1): L^{2} \rightarrow L^{2} \text { for } z \in \partial D .
$$

As in [ReSi, Lemma 13.1], we notice that for any $\delta \geqq 0:\langle x\rangle^{\delta}\left(Q_{\theta}^{i}-h^{-1} z\right)^{-1}\langle x\rangle^{-\delta}$ $=\mathcal{O}(1): L^{2} \rightarrow L^{2}$ uniformly for $z \in \partial D$. There follows:

$$
\begin{aligned}
& \left(P_{\theta}-z\right)^{-1} W J_{i}\left(P_{\theta}^{i}-z\right)^{-1} J_{i} W U_{\theta} \varphi_{\alpha} \\
& =h^{-1} U\left(Q_{\theta}-h^{-1} z\right)^{-1} W\left(h^{1 / 2} x\right) J_{i}\left(h^{1 / 2} x\right)\langle x\rangle^{-3} \\
& \quad \times h^{-1}\langle x\rangle^{3}\left(Q_{\theta}^{i}-h^{-1}\right)^{-1}\langle x\rangle^{-3} \cdot W\left(h^{1 / 2} x\right) J_{i}\left(h^{1 / 2} x\right)\langle x\rangle^{-3} \\
& \quad \times\langle x\rangle^{6} U^{-1} U_{\theta} \varphi_{\alpha}=\mathcal{O}(h) \text { in } L^{2} .
\end{aligned}
$$

The non-trapping condition (1.2) shows [BrCoDu, Lemma 2.5] that $\left(P_{\theta}^{e}-z\right)^{-1}$ $=\mathcal{O}(1): L^{2}\left(\operatorname{supp} J_{e}\right) \rightarrow L^{2}\left(\operatorname{supp} J_{e}\right)$ uniformly for $z \in \partial D$. As $U_{\theta} \varphi_{\alpha}$ decays exponentially outside the origin, we get:

$$
J_{e}\left(P_{\theta}^{e}-z\right)^{-1} J_{e} W U_{\theta} \varphi_{\alpha}=\mathcal{O}\left(e^{-\delta / h}\right) \text { in } L^{2}(\delta>0) .
$$

In the same way:

$$
h^{2} e^{-2 \theta}\left(P_{\theta}-z\right)^{-1}\left[\Delta, J_{e}\right]\left(P_{\theta}^{e}-z\right)^{-1} J_{e} W U_{\theta} \varphi_{\alpha}=\mathcal{O}\left(e^{-\delta / h}\right) \text { in } L^{2}
$$

[here we also use the fact that $\left(P_{\theta}^{e}-z\right)^{-1}=\mathcal{O}(1): L^{2}\left(\operatorname{supp} J_{e}\right) \rightarrow H^{2}\left(\operatorname{supp} J_{e}\right)$ for $z \in \partial D]$. We eventually get, by similar arguments:

$$
\begin{aligned}
& h^{2} e^{-2 \theta}\left(P_{\theta}-z\right)^{-1}\left[\Delta, J_{i}\right]\left(P_{\theta}^{i}-z\right)^{-1} J_{i} W U_{\theta} \varphi_{\alpha} \\
& =h^{-1} e^{-2 \theta} U\left(Q_{\theta}-h^{-1} z\right)^{-1}\left[\Delta, J_{i}\left(h^{1 / 2} x\right)\right]\left(Q_{\theta}^{i}-h^{-1} z\right)^{-1} \\
& \quad \times J_{i}\left(h^{1 / 2} x\right) W\left(h^{1 / 2} x\right)\langle x\rangle^{-3}\langle x\rangle^{3} U^{-1} U_{\theta} \varphi_{\alpha}=\mathcal{O}\left(h^{1 / 2}\right) \text { in } L^{2} .
\end{aligned}
$$

Summing up inequalities (1.17) to (1.21) we get by substitution in (1.15):

$$
\left(P_{\theta}-z\right)^{-1} W U_{\theta} \varphi_{\alpha}=\mathcal{O}\left(h^{1 / 2}\right) \text { in } L^{2}
$$

and (1.14) gives by integration on $\partial D$ :

$$
w_{\alpha}=U_{\theta} \varphi_{\alpha}+\mathcal{O}\left(h^{1 / 2}\right),
$$

which shows the first part of the lemma. The same procedure leads to:

$$
\Pi^{*} U_{\bar{\theta}} \varphi_{\beta}^{*}=U_{\bar{\theta}} \varphi_{\beta}^{*}+\mathcal{O}\left(h^{1 / 2}\right) \text { in } L^{2}
$$

To show (1.13) we follow the argument of [Sj, Sect. 4]. First we show, using the orthogonality conditions between $\operatorname{Ker} \Pi$ and $G^{*}, \operatorname{Ker} \Pi^{*}$ and $G$ :

We compute:

$$
\left(P_{\theta} w_{\alpha} \mid w_{\beta}^{*}\right)=\lambda_{0}(h) \delta_{\alpha \beta}+\left(W U_{\theta} \varphi_{\alpha} \mid w_{\beta}^{*}\right) \text {. }
$$

$$
\begin{aligned}
w_{\beta}^{*}-U_{\bar{\theta}} \varphi_{\beta}^{*} & =\left(w_{\beta}^{*}-\Pi^{*} U_{\bar{\theta}} \varphi_{\beta}^{*}\right)+\left(\Pi^{*} U_{\bar{\theta}} \varphi_{\beta}^{*}-U_{\bar{\theta}} \varphi_{\beta}^{*}\right) \\
& +\left(w_{\beta}^{*}-\Pi^{*} U_{\bar{\theta}} \varphi_{\beta}^{*}\right)+\mathcal{O}\left(h^{1 / 2}\right) \text { in } L^{2}
\end{aligned}
$$


Then we get:

$$
\left(w_{\alpha} \mid \Pi^{*} U_{\bar{\theta}} \varphi_{\beta}^{*}\right)=\delta_{\alpha \beta}+\left(w_{\alpha}-U_{\theta} \varphi_{\alpha} \mid U_{\bar{\theta}} \varphi_{\beta}^{*}-\Pi^{*} U_{\bar{\theta}} \varphi_{\beta}^{*}\right)=\delta_{\alpha \beta}+\mathcal{O}(h)
$$

by (1.12) and (1.22). There follows:

$$
\Pi^{*} U_{\bar{\theta}} \varphi_{\beta}^{*}-w_{\beta}^{*}=\mathcal{O}(h), \quad \text { whence: } \quad w_{\beta}^{*}-U_{\bar{\theta}} \varphi_{\beta}^{*}=\mathcal{O}\left(h^{1 / 2}\right) .
$$

So

$$
\left(W U_{\beta} \varphi_{\alpha} \mid w_{\beta}^{*}\right)=\left(W U_{\theta} \varphi_{\alpha} \mid U_{\bar{\theta}} \varphi_{\beta}^{*}\right)+\left(W U_{\theta} \varphi_{\alpha} \mid w_{\beta}^{*}-U_{\bar{\theta}} \varphi_{\beta}^{*}\right)
$$

As $W U_{\theta} \varphi_{\alpha}=\mathcal{O}\left(h^{3 / 2}\right)$ in $L^{2}$, we get the lemma.

End of the Proof of Lemma 1.2. There follows from (1.7) that $M$ has $q_{0}$ eigenvalues close to $\lambda_{0}(h)$ (counted with multiplicity); if we set $M-z=h\left(M^{\prime}-z^{\prime}\right)$, then

$$
(M-z)^{-1}=\frac{1}{h \operatorname{det}\left(M^{\prime}-z^{\prime}\right)}\left(M^{\prime}-z^{\prime}\right)^{*},
$$

where $\left(M^{\prime}-z^{\prime}\right)^{\prime \prime}=\mathcal{O}(1)$ by (1.13) is the transposed matrix of the cofactors of $M^{\prime}-z^{\prime}$. Let $\lambda_{1}(h)=\lambda(h), \lambda_{2}(h), \ldots, \lambda_{q_{0}}(h)$ be the eigenvalues of $M$, counted with multiplicity. We have:

$$
\operatorname{det}(M-z)=(\lambda(h)-z)\left(\lambda_{2}(h)-z\right) \ldots\left(\lambda_{q_{0}}(h)-z\right)
$$

and for $z \in \partial \widetilde{D}$, the relation (1.9) shows: $\left|\operatorname{det}\left(M^{\prime}-z^{\prime}\right)\right| \geqq C h^{(N-1) q_{0}}$. Then we get: $(M-z)^{-1}=\mathcal{O}\left(h^{(1-N) q_{0}-1}\right)$. The estimates $(1.12)$ and $w_{\beta}^{*}=U_{\bar{\theta}} \varphi_{\beta}^{*}+\mathcal{O}\left(h^{1 / 2}\right)$ then lead to:

$$
\Pi\left(P_{\theta}-z\right)^{-1} \Pi=\mathcal{O}\left(h^{(1-N) q_{0}-1}\right)
$$

provided $\varphi_{\alpha}$ and $\varphi_{\beta}^{*}$ are normalized. Substituting (1.11) and (1.23) in (1.10) we eventually get the lemma.

We now perturb $P$, changing $V$ in:

$$
\widetilde{V}(x, h)=V(x)+\Delta V(x, h)
$$

where $\Delta V$ is a finite sum of potentials having the form:

$$
\Delta V_{l, m}(x, h)=h^{l} q_{2 m}(x, h) e^{-\frac{x^{2}}{2}},
$$

where $l, m \in \mathbb{N}^{*}, l+m=v=$ const and $q_{2 m}$ is a polynomial in $x$ homogeneous of degree $2 m$ whose coefficients are bounded functions of $h$. We consider $\widetilde{P}_{\theta}=P_{\theta}+\Delta V\left(e^{\theta} x\right)$ as an analytic family of type $(A)$ on $L^{2}$ for $|\operatorname{Im} \theta|$ small enough. We get:

$$
\Delta V\left(e^{\theta} x\right)=\widetilde{P}_{\theta}-P_{\theta}=\mathcal{O}\left(h^{l}\right): L^{2} \rightarrow L^{2} .
$$

By Lemma 1.2, if $l>N_{1}$, the Neumann series:

$$
\sum_{k=0}^{+\infty}\left(P_{\theta}-z\right)^{-1}\left(\left(\widetilde{P}_{\theta}-P_{\theta}\right)\left(P_{\theta}-z\right)^{-1}\right)^{k}
$$

converging for $h>0$ small enough defines $\left(\widetilde{P}_{\theta}-z\right)^{-1}$ as a bounded operator on $L^{2}$ for all $z \in \partial D\left(\lambda(h), \delta h^{N}\right)$. Furthermore:

$$
\begin{gathered}
\left(\widetilde{P}_{\theta}-z\right)^{-1}=\mathcal{O}\left(h^{-N_{1}}\right): L^{2} \rightarrow L^{2}, \\
\left(\widetilde{P}_{\theta}-z\right)^{-1}-\left(P_{\theta}-z\right)^{-1}=\mathcal{O}\left(h^{l-2 N_{1}}\right): L^{2} \rightarrow L^{2} .
\end{gathered}
$$


If $l>2 N_{1}$, the spectral projector:

$$
\tilde{\Pi}=\frac{1}{2 i \pi} \int_{\partial \tilde{D}}\left(z-\widetilde{P}_{\theta}\right)^{-1} d z
$$

where $\tilde{D}=D\left(\lambda(h), \delta h^{N}\right)$, associated to the resonances of $\widetilde{P}_{\theta}$ in $\widetilde{D}$, is close in $\mathscr{L}\left(L^{2}\right)$ to the corresponding projector for $P_{\theta}$. In particular, if $q$ is the (algebraic) multiplicity of $\lambda(h)$ and $\left(\Psi_{\beta}\right)$ denotes an orthonormal basis of $F=F_{\lambda}$, then there are exactly $q$ resonances in $\widetilde{D}$ for $\widetilde{P}_{\theta}$ and $\widetilde{F}=\widetilde{\Pi}\left(L^{2}\right)$ is a characteristic subspace of $\operatorname{dim} q$, a basis of which is given by:

$$
v_{\beta}=\tilde{\Pi}\left(\Psi_{\beta}\right), \quad \beta=1,2, \ldots, q .
$$

In the same way we consider, for $z \in \partial \widetilde{D}^{*}=\partial D\left(\bar{\lambda}(h), \delta h^{N}\right)$ the operator $\left(\widetilde{P}_{\bar{\theta}}-z\right)^{-1}: L^{2} \rightarrow L^{2}$ and the spectral projector:

$$
\tilde{\Pi}^{*}=\frac{1}{2 i \pi} \int_{\partial \tilde{D}^{*}}\left(z-\widetilde{P}_{\bar{\theta}}\right)^{-1} d z
$$

associated to the incoming resonances of $\widetilde{P}$ in $\widetilde{D}^{*}$. The subspace $\widetilde{F}^{*}=\tilde{\Pi}^{*}\left(L^{2}\right)$ is a characteristic subspace of $\operatorname{dim} q$, dual of the latter, a basis of which is given by the $\widetilde{\Pi}^{*}\left(\Psi_{\gamma}^{*}\right), \beta=1,2, \ldots, q$, where $\left(\Psi_{\beta}^{*}\right)$ is the dual basis of the $\left(\Psi_{\beta}\right)$. Let $v_{\beta}^{*} \in \widetilde{F}_{\lambda}^{*}$ be the dual basis of the $\left(v_{\beta}\right)$. We then assume that $\lambda(h)$ is a semi-simple eigenvalue of $P_{\theta}$, i.e.

$$
\left(P_{\theta} \Psi_{\beta} \mid \Psi_{\gamma}^{*}\right)=\lambda(h) \delta_{\beta \gamma}
$$

Then the matrix of $\widetilde{P}_{\theta}$ in the basis $\left(v_{\beta}\right)$ is given by $\left(\widetilde{P}_{\theta} v_{\beta} \mid v_{\gamma}^{*}\right)=\left(\widetilde{P}_{\theta} \Psi_{\beta} \mid v_{\gamma}^{*}\right)=\lambda(h) \delta_{\beta \gamma}$ $+\left(\Delta V \Psi_{\beta} \mid v_{\gamma}^{*}\right)$. As in [Sj] and the proof of Lemma 1.3 we get:

$$
\begin{aligned}
v_{\gamma}^{*}-\Psi_{\gamma}^{*} & =\left(v_{\gamma}^{*}-\tilde{\Pi}^{*} \Psi_{\gamma}^{*}\right)+\left(\tilde{\Pi}^{*} \Psi_{\gamma}^{*}-\Psi_{\gamma}^{*}\right) \\
& =\mathcal{O}\left(h^{2(l+m)-2 N_{1}}\right)+\mathcal{O}\left(h^{l+m-N_{1}}\right) \text { in } L^{2} .
\end{aligned}
$$

We then have to estimate $\Delta V \Psi_{\beta}$ in $L^{2}$. As $F C G=\Pi\left(L^{2}\right)$, there exists constants $a_{\alpha \beta} \in \mathbb{C}$ with $a_{\alpha \beta}=\mathcal{O}(1)$ such that $\Psi_{\beta}=\sum_{|\alpha|=\sigma} a_{\alpha \beta} w_{\alpha}$, where $\left(w_{\alpha}\right)$ is the basis of $G$ introduced in Lemma 1.2. By (1.15):

$$
\begin{aligned}
\Delta V \Psi_{\beta}= & \sum_{|\alpha|=\sigma} a_{\alpha \beta} \Delta V U_{\theta} \varphi_{\alpha} \\
& +\sum_{|\alpha|=\sigma} a_{\alpha \beta} \int_{\partial D}\left(\lambda_{0}(h)-z\right)^{-1} \Delta V\left(P_{\theta}-z\right)^{-1} W U_{\theta} \varphi_{\alpha} d z
\end{aligned}
$$

and by the explicit form of $\Delta V$ and $\varphi_{\alpha}$ we easily get $\Delta V U_{\theta} \varphi_{\alpha}=\mathcal{O}\left(h^{l+m}\right)$ in $L^{2}$. For the complementary term, we again consider resolvent equation (1.16) and analyse each of the terms as in the proof of Lemma 1.3. Then it is clear that $\Delta V\left(P_{\theta}-z\right)^{-1} W U_{\theta} \varphi_{\alpha}$ $=\mathcal{O}\left(h^{l+m+\frac{1}{2}}\right)$ in $L^{2}$, which together with (1.25) shows that $\left(\Delta V \Psi_{\beta} \mid v_{\gamma}^{*}\right)$ $=\left(\Delta V \Psi_{\beta} \mid \Psi_{\gamma}^{*}\right)+\mathcal{O}\left(h^{2(l+m)-N_{1}}\right)$. We eventually proved the:

Proposition 1.4. Under hypothesis (1.1) to (1.4), (1.9) and (1.24) let $\tilde{M}=\left(\widetilde{P}_{\theta} v_{\beta} \mid v_{\gamma}^{*}\right)$ the matrix of $\widetilde{P}_{\theta} \uparrow \tilde{F}$ in the basis $\left(v_{\beta}\right)$. Then if $l$ is large enough and $l+m=v=$ const we have:

$$
\left(\widetilde{P}_{\theta} v_{\beta} \mid v_{\gamma}^{*}\right)=\lambda(h) \delta_{\alpha \beta}+\left(\Delta V \Psi_{\beta} \mid \Psi_{\gamma}^{*}\right)+\mathcal{O}\left(h^{2(l+m)-N_{1}}\right)
$$

uniformly for $h>0$ small enough, with $N_{1} \geqq 1$ independent of $l$ and $m$. 


\section{A Simple Case in Two Dimensions}

Here we consider the hamiltonian $P_{0}=-h^{2} \Delta-|x|^{2}$. Constructions of Part 1 apply to this situation which is also treated in [Sj]. We get the following:

Theorem 2.1 For any integer $q>0$, there exists a compact perturbation $\Delta V(x, h)$ of $P_{\theta}=-h^{2} \Delta-|x|^{2}$ such that the resolvent $(\widetilde{P}-z)^{-1}$ of $\widetilde{P}=P_{0}+\Delta V(x, h)$ for $\operatorname{Im} z>0$, extends across the real axis for $z$ close to 0 (in the sense of analytic dilations), as a meromorphic function which has a pole of order $q$, for any $h>0$ small enough, in a neighborhood of the semi-simple resonance $\lambda_{0}(h)=-2 i h q$ of $P_{0}$.

With the notations of Proposition 1.4, $\Psi_{\beta}=\varphi_{\beta}$ [formula (1.6)], and $q=q_{0}$ $=\sigma+1$, we have:

$$
\left(\widetilde{P}_{\theta} v_{\alpha} \mid v_{\beta}^{*}\right)=\lambda_{\theta}(h) \delta_{\alpha \beta}+\left(\Delta V \varphi_{\alpha} \mid \varphi_{\beta}^{*}\right)+\mathcal{O}\left(h^{2(l+m)-1}\right)
$$

for $l, m \in \mathbb{N}^{*}, l+m=v=$ const, $|\alpha|=|\beta|=\sigma \in \mathbb{N}^{*}$ and $\lambda_{0}(h)=-i h(2 \sigma+2)$. A simple computation as in $[\mathrm{Sj}]$ shows that

$$
\left(\Delta V \varphi_{\alpha} \mid \varphi_{\beta}^{*}\right)=h^{v} \sum_{m} i^{m}\left(\int_{\mathbb{R}^{2}} e^{-x^{2}} q_{2 m}(x, 0) \Phi_{\alpha}(x) \Phi_{\beta}(x) d x+\mathcal{O}(h)\right),
$$

where the $\Phi_{\alpha}$ are the usual Hermite polynomials (the $\widetilde{\Phi}_{\alpha}$ are obtained from the $\Phi_{\alpha}$ by a rotation of $\pi / 4$ in the complex domain).

The first step in our construction consists in obtaining any element of the space $M_{q}^{*}(\mathbb{C})$ of complex symmetric matrices of order $q$ as a finite sum:

$$
\left(\sum_{m}^{i^{m}}\left(\int e^{-x^{2}} q_{2 m}(x, 0) \Phi_{\alpha}(x) \Phi_{\beta}(x) d x\right)_{|\alpha|=|\beta|=\sigma} .\right.
$$

Elementary properties of Hermite functions show the following:

Lemma 2.2. Let $L_{+}^{2}$ be the closed linear subspace in $L^{2}\left(\mathbb{R}^{2} ; e^{-x^{2}} d x\right)$ of even functions defined on $\mathbb{R}^{2}$. Then for any $\sigma \in \mathbb{N}$, the family $\left(\Phi_{\alpha} \Phi_{\beta}\right)_{|\alpha|=|\beta|=\sigma}$ spans a linear subspace $E$ of dimension $q(q+1) / 2$ in $L_{+}^{2}$ (we identify $\Phi_{\alpha} \Phi_{\beta}$ with $\Phi_{\beta} \Phi_{\alpha}$ ).

Let us consider the linear subspaces $\mathscr{D}_{1}$ (respectively $\mathscr{D}_{2}$ ) in $L_{+}^{2}$ spanned by homogeneous polynomials $q_{2 m}$ with real coefficients (also depending on $h$ ) of degree $2 m\left(m \in \mathbb{N}^{*}\right)$ with even (respectively odd) $m$. We have:

Proposition 2.3. For $s=1,2, \mathscr{D}_{s}$ is a dense subspace of $L_{+}^{2}$.

By Fourier transform in $\mathbb{R}$ and a Paley-Wiener type argument we can prove the following:

Lemma 2.4. The set of homogeneous polynomials $\left(x^{4 m}\right)_{m \geqq 1}$ (respectively $\left.\left(x^{4 m+2}\right)_{m \geqq 0}\right)$ is dense in $L^{2}\left(\mathbb{R}^{+} ; e^{-x^{2}} d x\right)$.

Proof of the Proposition. First we notice that Lemma 2.4 still holds when reinforcing a little the $L^{2}$ norm, e.g. replacing $L^{2}\left(\mathbb{R}^{+} ; e^{-x^{2}} d x\right)$ by $L^{2}\left(\mathbb{R}^{+} ; e^{-3 x^{2} / 4} d x\right)$. We consider the orthogonal projectors in $L_{+}^{2}: f(x) \rightarrow \frac{1}{2}\left(f\left(x_{1},-x_{2}\right)+f\left(x_{1}, x_{2}\right)\right)$ and $f(x) \rightarrow \frac{1}{2}\left(-f\left(x_{1},-x_{2}\right)+f\left(x_{1}, x_{2}\right)\right)$, with range $L_{++}^{2}$ and $L_{--}^{2}$. For $\varphi \in L_{++}^{2}$ $\cup L_{--}^{2}$ :

$$
\|\varphi\|_{L^{2}+}=2\|\varphi\|_{L^{2}\left(\mathbb{R}^{+} \times \mathbb{R}^{+} ; \exp \left(-x^{2}\right) d x\right)} .
$$

Let $\Omega=\mathbb{R}^{2} \backslash\left\{x_{1} x_{2}=0\right\}$. The proposition follows if we can approximate in $L^{2}\left(\mathbb{R}^{2} ; e^{-3 x^{2} / 4} d x\right)$ any function $\varphi \in C_{0}^{\infty}(\Omega)$ by polynomials in $\mathscr{D}_{s}, s=1,2$. If 
$\varphi \in C_{0}^{\infty}(\Omega)$, then $\Psi(x)=x_{2}^{-2} \varphi(x) \in C_{0}^{\infty}(\Omega)$. Then there suffices to show the proposition only for $\mathscr{D}_{1}$. As the set of polynomials $x^{4 m}$ is a dense subspace of $L^{2}\left(\mathbb{R}^{2} ; e^{-3 x^{2} / 4} d x\right)$, we get an hilbertian basis of $L^{2}\left(\mathbb{R}^{+} \times \mathbb{R}^{+} ; e^{-3 x^{2} / 4} d x\right)$ consisting in elements of $\mathscr{D}_{1}$ by the usual Gram-Schmidt procedure. Let $\varphi \in C_{0}^{\infty}\left(\mathbb{R}^{+} \times \mathbb{R}^{+}\right)$. Then:

$$
\varphi(x)=\sum_{j=0}^{+\infty} c_{j} e_{j}\left(x_{1}^{4}, x_{2}^{4}\right)
$$

with convergence in $L^{2}\left(\mathbb{R}^{+} \times \mathbb{R}^{+} ; e^{-3 x^{2} / 4} d x\right)$ (the $e_{j}$ being polynomials with real coefficients). If $\varphi \in C_{0}^{\infty}(\Omega) \cap L_{++}^{2}$, relations (2-5) and (2-6) show that $\varphi$ is the limit in $L_{+}^{2}$ of a sequence of elements of $\mathscr{D}_{1}$. Finally, let us consider $\varphi \in C_{0}^{\infty}(\Omega) \cap L_{-}^{2}$. Then:

$$
\Psi(x)=x_{1}^{-1} x_{2}^{-3} \varphi\left(x_{1}, x_{2}\right) \in L_{++}^{2},
$$

and:

$$
\varphi(x)=x_{1} x_{2}^{3} \sum_{j=0}^{+\infty} c_{j} e_{j}\left(x_{1}^{4}, x_{2}^{4}\right)
$$

with convergence in $L^{2}\left(\mathbb{R}^{2} ; e^{-x^{2}} d x\right)$, which finishes the proof.

The mapping $f: L_{+}^{2} \rightarrow M_{q}^{+}(\mathbb{R})$ (space of real symmetric matrices of order $q$ ) defined by:

$$
f(\varphi)=\left(a_{\alpha \beta}(\varphi)\right)_{|\alpha|=|\beta|=\sigma}=\left(\int e^{-x^{2}} \Phi_{\alpha}(x) \Phi_{\beta}(x) \varphi(x)_{|\alpha|=|\beta|=\sigma}\right.
$$

is clearly an isomorphism from $E$ to $M_{q}^{+}(\mathbb{R})$ (Gram matrix). Let $\Pi_{+}: L_{+}^{2} \rightarrow E$ the orthogonal projection on $E$. As $E$ is finite dimensional, Proposition 2.3 shows that $\Pi_{+}\left(\mathscr{D}_{s}\right)=E(s=1,2)$ and $f \circ \Pi_{+}$is onto. We denote:

$$
\mathscr{M}_{0}: \mathscr{D}_{1} \oplus \mathscr{D}_{2} \rightarrow M_{q}^{+}(\mathbb{C})
$$

the mapping defined by $\mathscr{M}_{0}\left(Q_{1}, Q_{2}\right)=f \circ \Pi_{+}\left(Q_{1}\right)+i f \circ \Pi_{+}\left(Q_{2}\right)$. Then $\mathscr{M}_{0}$ is oneto-one from $\mathscr{D}_{1} /\left(E^{\perp} \cap \mathscr{D}_{1}\right) \oplus \mathscr{D}_{2} /\left(E^{\perp} \cap \mathscr{D}_{2}\right)$ to $M_{q}^{+}(\mathbb{C})$. Choose among all the representatives a subspace $\mathscr{E}$ of $\mathscr{D}_{1} \oplus \mathscr{D}_{2}$ spanned by a family of $q(q+1)$ polynomials whose degrees are minimal. If $Q_{s}=\sum q_{2 m}(x) \in \mathscr{D}_{s}$, define $\widetilde{Q}_{s}=\sum h^{l} q_{2 m}(x)$ with $l, m \in \mathbb{N}^{*}$ and $v=l+m=$ const. Let $\mathscr{D}_{s}$ the space corresponding to $\mathscr{D}_{s}, \widetilde{E}$ the space corresponding to $\mathscr{E}$ (giving minimal $v$ ) and $\widetilde{M}_{0}: \mathscr{E} \rightarrow M_{q}^{+}(\mathbb{C})$ the isomorphism defined by $\tilde{\mathscr{M}}_{0}\left(\tilde{Q}_{1}, \widetilde{Q}_{2}\right)=\mathscr{M}_{0}\left(Q_{1}, Q_{2}\right)$. Using the fact that $i^{m}$ is imaginary if $m$ is odd, real if $m$ is even, formulae (2.1) and (2.2) then give the:

Proposition 2.5. Let:

$$
\widetilde{\mathscr{D}}_{s}=\left\{\widetilde{Q}_{s}=\sum_{l+m=v} h^{l} q_{2 m}(x, h) \mid v \geqq 2, m \equiv s+1 \bmod 2\right\} .
$$

For $n=2$, consider perturbations of the hamiltonian $P_{0}=-h^{2} \Delta-|x|^{2}$ of the form $\Delta V=\left(\widetilde{Q}_{1}+\widetilde{Q}_{2}\right) e^{-x^{2} / 2}$ with $\widetilde{Q}_{s} \in \widetilde{D}_{s}$. Then there exists a $\mathbb{R}$-linear map $\left(\widetilde{Q}_{1}, \widetilde{Q}_{2}\right) \rightarrow \tilde{M}_{0}\left(\widetilde{Q}_{1}, \widetilde{Q}_{2}\right)$ which realizes an isomorphism from a linear subspace $\widetilde{E}$ of $\mathscr{D}_{1} \oplus \mathscr{D}_{2}$ in $M_{q}^{+}(\mathbb{C})$ such that the matrix $\widetilde{P}\lceil\widetilde{F}$ defined in Proposition (1.4) is of the form:

$$
\begin{aligned}
\tilde{\mathscr{M}} & =\lambda_{0}(h) I d+h^{v}\left(\widetilde{\mathscr{M}}_{0}\left(\widetilde{Q}_{1}, \widetilde{Q}_{2}\right)+\mathcal{O}(h)\right) \\
& =\lambda_{0}(h) I d+h^{v} \mathscr{M}\left(\widetilde{Q}_{1}, \widetilde{Q}_{2}, h\right) \quad(v \geqq 2) .
\end{aligned}
$$

The next step consists in noticing that any complex nilpotent matrix is congruent to a symmetric matrix, and in establishing a geometrical transversality lemma to absorb the $\mathcal{O}(h)$ term in $(2.2)$. Let $M_{q}(\mathbb{C})$ the space of all complex matrices of order $q$ and $\mathscr{H} \subset M_{q}(\mathbb{C})$ the complex manifold of matrices $M$ nilpotent exactly at order $q$ (i.e. $M^{q}=0, M^{q-1} \neq 0$ ). 
Proposition 2.6. For any $M \in \mathscr{H}$ we have

$$
q=\operatorname{codim}_{M_{q}(\mathbb{C})} T_{M} \mathscr{H}=\operatorname{codim}_{M_{q}^{+}(\mathbb{C})} T_{M^{\prime}} \mathscr{H} \cap M_{q}^{+}(\mathbb{C}) .
$$

Proof. The first equality in (2.7) is classical; for instance we may, without loss of generality, assume that $M$ is given by its real Jordan form $J$; in a neighborhood of $J$ it is easily seen that the manifold $\mathscr{H}$ is given by:

where

$$
f_{1}(N)=\ldots=f_{q}(N)=0,
$$

$$
d f_{j}(\delta N)=\sum_{i=1}^{q-j+1} a_{i+j-1, i}, \quad \delta N=\left(a_{i j}\right)_{1 \leqq i, j \leqq q} \in T_{J} \mathscr{H}
$$

is a system of $q$ linearly independent forms on $\mathbb{C}$. On the other hand we know (see e.g. Gantmacher [G]) that any $M \in \mathscr{H}$ is congruent to a symmetric complex matrix $K \in M_{q}^{+}(\mathbb{C}) \cap \mathscr{H}$. We begin with computing $T_{M} \mathscr{H}$ intrinsically. If $A \in M_{q}(\mathbb{C})$ we have

$$
(M+\varepsilon A)^{q}=\varepsilon B(A)+\mathcal{O}\left(\varepsilon^{2}\right)
$$

where

$$
B(A)=M^{q-1} A+M^{q-2} A M+\ldots+A M^{q-1} .
$$

Lemma 2.7. The map $\theta: M_{q}(\mathbb{C}) \ni A \rightarrow B(A)$ has kernel $T_{M} \mathscr{H}$ and is surjective onto $(\operatorname{Ad} M)^{-1}(0)=\left\{B \in M_{q}(\mathbb{C}) ;[\mathrm{B}, \mathrm{M}]=0\right\}$.

Proof. First notice that for any $A \in M_{q}(\mathbb{C})$ we have $[B(A), M]=0$. Next observe that $(\operatorname{Ad} M)^{-1}(0)$ is a subspace of dimension $q\left[\right.$ compute $B \in(\operatorname{Ad} M)^{-1}(0)$ in a basis $\left(e_{j}\right)_{1 \leqq j \leqq q}$ such that $e_{i}=M^{q-i} e_{q}$ with $\left.M^{q-1} e_{q} \neq 0\right]$. Let us show that $\operatorname{codim}_{M_{q}(\mathbb{C})} \operatorname{Ker} \theta=q . B=B(A)$ and $\left(e_{j}\right)_{1 \leqq j \leqq q}$ is defined as above, we get

$$
B e_{j}=\sum_{k=1}^{j} M^{q-j-k-1} A e_{k}, \quad j=1, \ldots, q .
$$

Let $A=\left(a_{i j}\right)_{1 \leqq i, j \leqq q}$ in the basis $\left(e_{k}\right)$. The condition $B(A)=0$ gives $q$ independent relations on the $a_{i j}$ :

$$
\sum_{k=1}^{j} a_{q-j+k, k}=0 \quad j=1, \ldots, q
$$

(first component of $B e_{j}=0$ ). Then we have $\operatorname{codim} \operatorname{Ker} \theta \geqq q$. On the other hand $\operatorname{Ker} \theta \supset T_{M} \mathscr{H}$, and the latter space has codimension $q$; thus $\operatorname{Ker} \theta=T_{M} \mathscr{H}$ and the lemma is proved.

We then work in a neighborhood of $M=K$. Up to a diffeomorphism the relations (2.8) define $\mathscr{H}$ near $K$. By Lemma 2.5 , we can identify $\theta$ with the differential of $A \rightarrow\left(f_{1}(A), \ldots, f_{q}(A)\right)$. On the other hand, we check that if $B \in(\operatorname{Ad} K)^{-1}(0)$, then ${ }^{t} B=B$. Let $\mathscr{H}^{+}=\mathscr{H} \cap M_{q}^{+}(\mathbb{C})$. Then near $K$,

$$
\mathscr{H}^{+}=\left\{N \in M_{q}^{+}(\mathbb{C}): f_{1}(N)=\ldots=f_{q}(N)=0\right\} .
$$

Then there suffices to show that $d f_{1}, \ldots, d f_{q}$ (at $K$ ) are linearly independent differentials on $M_{q}^{+}(\mathbb{C})$. Let $B \in(\operatorname{Ad} K)^{-1}(0)$. We have seen that $B$ is symmetric and by Lemma 2.5 there exists $A \in M_{q}(\mathbb{C})$ such that $B=B(A)$. As $B\left({ }^{t} A\right)={ }^{t} B(A)$ we find $B\left(\frac{1}{2}^{2} A+\frac{1}{2}^{t} A\right)=\frac{1}{2}^{2} B+\frac{1}{2}^{t} B=B$. Then $\theta\left\lceil M_{q}^{+}(\mathbb{C})\right.$ is onto, which gives the desired result. 
There easily follows the:

Corollary 2.8. Let $K \in M_{q}^{+}(\mathbb{C}) \cap \mathscr{H}$. Then there exists in a neighborhood of $K$ an affine subspace $\mathscr{V}_{0}=M_{q}^{+}$of dimension $q$ whose intersection with $\mathscr{H}$ is transversal, i.e. $\mathscr{V}_{0} \oplus\left(T_{K} \mathscr{H} \cap M_{q}^{+}(\mathbb{C})\right)=M_{q}^{+}(\mathbb{C})$.

We conclude to the existence of multiple poles for the resolvent of $P\lceil\tilde{F}$ as $h \rightarrow 0$ as in [Sj]. Let $\mathscr{V}_{0}^{*}=\mathscr{M}_{0}^{-1}\left(\mathscr{V}_{0}\right)$. Thus $\mathscr{V}_{0}^{*}$ is an affine-linear subspace of dimension $q$ containing $\mathscr{M}_{0}^{-1}(K)$. As in [Sj] we verify that the maps $\mathscr{M}_{0}$ and $\mathscr{M}$ are analytic with respect to $\widetilde{Q}_{1}$ and $\widetilde{Q}_{2}$ and that their differentials satisfy:

$$
d \mathscr{M}=d \mathscr{M}_{0}+\mathcal{O}(h) \text {. }
$$

Let $\mathscr{V}_{h}$ the image of $\mathscr{V}_{0}^{*}$ through $\mathscr{M}$. Then $\mathscr{V}_{h}$ is an analytic submanifold of real dimension $2 q$ which differs in a neighborhood of $K$ from the manifold $\mathscr{V}_{0}$ by a quantity $\mathcal{O}_{h}$ (for the $\mathscr{C}^{\infty}$ topology). In particular, if $h>0$ is small enough $\mathscr{V}_{h}$ intersects transversally $\mathscr{H}$ in a neighborhood of $K$ whose size is of order $h$. Thus we proved that there exists an affine-linear submanifold $\mathscr{V}_{0}^{*} e^{-x^{2} / 2} C \mathscr{E} e^{-x^{2} / 2}$ (with obvious notations) of real dimension $2 q$ consisting in potentials $\Delta V(h)=\Delta V(x, h)$ $=\left(\widetilde{Q}_{1}+\widetilde{Q}_{2}\right) e^{-x^{2} / 2}$, and a real analytic map $h \rightarrow \Delta V(h) \in \mathscr{V}_{0}^{*} e^{-x^{2} / 2}$ defined for $h>0$ small enough such that $\tilde{M}$ has a nilpotent part to the order $q$. This achieves the proof of Theorem 2.1 .

When trying to extend Theorem 2.1 to radial potentials verifying assumptions (1.1) to (1.4) and (1.2)', we encounter the following obstruction: the multiplicity $q$ of $\lambda(h)$ is generally equal to the dimension of the space of spherical harmonics associated to the orbital quantum number $\sigma$. For $n=2$ we then have $q=2$ for any $\sigma \geqq 1$ and the result of [Sj] cannot be improved in general. Note however that by choosing potentials which have a high contact order with $-|x|^{2}$ at $x=0$, it is still possible to construct perturbations $\Delta V$ which give rise to arbitrarily high order poles for the resolvent of $\widetilde{P}$. But such an extension is not very natural; the good generalization of our result occurs in dimension 3.

\section{Case of Radial Potentials in Dimension 3}

For a radial symmetric potential, the states corresponding to a same "energy" level $\lambda_{0}(h)=-i h(2 \sigma+n)$ of the hamiltonian $P_{0}=-h^{2} \Delta+\frac{1}{2} V(0) \cdot x . x$ generally split according to the orbital quantum number (quantization of orbital momentum); these states only can be perturbed by breaking the spherical symmetry to obtain degeneracies, for Theorem 2.1 does not directly extend to $P_{0}$ as soon as $n \geqq 3$. If $n=3$, the (geometric) multiplicity of a resonance $\lambda(h)$ of $P=-h^{2} \Delta+V$ close to $\lambda_{0}(h)$ [in the sense of $(1.7)]$ is equal to $2(\sigma-2 k)+1$, for $k=0,1, \ldots,[\sigma / 2]$.

(From the point of view of representation theory, we can say that the space of corresponding resonant functions (after dilation) is an irreducible component of $L^{2}\left(\mathbb{R}^{3}\right)$ under the action of the group $0^{+}(3)$, isomorphic to an eigenspace of the Laplace-Beltrami operator on $L^{2}\left(S^{2}\right)$, while the eigenspaces of $P_{0}$ split into several irreducible components. (For an introduction to problems relevant to representation theory in Quantum Mechanics, we refer e.g. to Mackey [M].)

In particular, for $k=0, \lambda(h)$ is the first resonance (state $s$ ) of the operator

$$
P(\sigma)=-h^{2}\left(\frac{\partial^{2}}{\partial r^{2}}+\frac{2}{r} \frac{\partial}{\partial r}-\frac{\sigma(\sigma-1)}{r^{2}}\right)+V\left(r^{2}\right),
$$


acting on the $r$ variable, where $x=r \omega, r>0, \omega \in S^{2}$ (of course, non-zero $k$ 's correspond to other values of $\sigma$ ). We are going to show that the algebraic multiplicity of the $\lambda(h)$ 's is equal to their geometric multiplicity (at least as $h$ tends to zero) or, in other terms, that the resolvent of $P$ has only simple poles.

Let us recall some properties of spherical harmonics. Let $\Delta_{\omega}$ be the Laplace operator on $S^{n-1}$. The eigenvalues of $-\Delta_{\omega}$ have the form $\lambda_{\sigma}=\sigma(\sigma+n-1)$ and their multiplicity is $d_{\sigma}=\left(\begin{array}{c}\sigma-n-1 \\ \sigma\end{array}\right)-\left(\begin{array}{c}\sigma-n-3 \\ \sigma-2\end{array}\right)$. Let $\mathscr{Y}_{\sigma}$ the corresponding eigenspace, then $\mathscr{Y}_{\sigma}$ is an irreducible component of $L^{2}\left(S^{n-1}\right)$ under the action of $0^{+}(n)$, isomorphic to the space of harmonic polynomials on $\mathbb{R}^{n}$ homogeneous of degree $\sigma$. If $n=3$ we have $d_{\sigma}=2 \sigma+1$ and

$$
\mathscr{Y}_{\sigma}=\operatorname{Vect}\left\{Y_{\sigma, \tau} \mid-\sigma \leqq \tau \leqq \sigma\right\} .
$$

Let $P=-h^{2} \Delta+V$ on $L^{2}\left(\mathbb{R}^{3}\right)$, where $V$ satisfies to hypotheses (1.1), (1.2), (1.3), and (1.4). $P$ is not essentially self-adjoint on $\mathscr{C}_{0}^{\infty}\left(\mathbb{R}^{3} \backslash 0\right)$, but we can consider its Friedrichs extension and it is easy to see that it defines a non-bounded operator on $L^{2}\left(\mathbb{R}^{3}\right)$ with domain $\mathscr{D}(P)=H^{2}\left(\mathbb{R}^{3}\right)$ : thus this extension is precisely the natural extension of $P$ from $\mathscr{C}_{0}^{\infty}\left(\mathbb{R}^{3}\right)$. In polar coordinates we have $P=\bigoplus_{\sigma=0}^{\infty} \bigoplus_{\tau=-\sigma}^{\tau=\sigma} P(\sigma)$, where $P(\sigma)$ is the self-adjoint extension from $\mathscr{C}_{0}^{\infty}\left(\mathbb{R}^{+}\right)$of operator (3.1), whose domain is contained in:

$$
H_{\sigma}^{1}\left(\mathbb{R}^{+}\right)=\left\{u \in L^{2}\left(\mathbb{R}^{+}, r^{2} d r\right) \mid \int_{0}^{\infty}\left(\left(1+\frac{\sigma(\sigma+1)}{r^{2}}\right)|u|^{2}+\left|\frac{\partial u}{\partial r}\right|^{2}\right) r^{2} d r<\infty\right\} .
$$

Then we get $\mathscr{D}(P(\sigma))=H_{0, \sigma}^{1}\left(\mathbb{R}^{+}\right) \cap \mathscr{H}_{\sigma}$ where $H_{0, \sigma}^{1}\left(\mathbb{R}^{+}\right)$is the closure of $\mathscr{C}_{0}^{\infty}\left(\mathbb{R}^{+}\right)$ in $H_{\sigma}^{1}\left(\mathbb{R}^{+}\right)$and:

$$
\begin{aligned}
\mathscr{H}_{\sigma} & =\left\{u \in L^{2}\left(\mathbb{R}^{+}, r^{2} d r\right) \mid P(\sigma) u \in L^{2}\left(\mathbb{R}^{+}, r^{2} d r\right)\right\} \\
& =\left\{u \in L^{2} \mid-h^{2} \Delta(\sigma) u \equiv-h^{2}\left(\frac{\partial^{2}}{\partial r^{2}}+\frac{2}{r} \frac{\partial}{\partial r}-\frac{\sigma(\sigma+1)}{r^{2}}\right) u \in L^{2}\right\} .
\end{aligned}
$$

We can recover $\mathscr{D}(P)$ by means of the formula:

$$
\begin{aligned}
\mathscr{D}(P)=\{ & u=\sum_{\sigma=0}^{+\infty} \sum_{\tau=-\sigma}^{\sigma} u_{\sigma \tau} Y_{\sigma \tau}\left|u_{\sigma \tau} \in \mathscr{D}(P(\sigma)) \forall \sigma, \tau\right| \\
& \left.\left.\sum_{\sigma=0}^{+\infty} \sum_{\tau=-\sigma}^{\sigma}\left\|u_{\sigma \tau}\right\|^{2} \widehat{H_{\sigma}^{1}\left(\mathbb{R}^{+}\right.}\right)<\infty \mid \sum_{\sigma=0}^{+\infty} \sum_{\tau=-\sigma}^{\sigma}\|P(\sigma) u\|_{L^{2}}^{2}<\infty\right\}
\end{aligned}
$$

(see e.g. [Ro]). We deduce:

$$
S p(P)=\bigcup_{\sigma=0}^{+\infty} S p(P(\sigma))
$$

[note that the (locally finite) union is generally disjoint, in an asymptotic sense that will become clear in the Appendix, which is not the case when $P(\sigma)$ is replaced by the hamiltonian $\widetilde{P}(\sigma)=-h^{2} \Delta(\sigma)-r^{2}$.

Let $U_{\theta}$ the operator of analytic dilations, and:

$$
P_{\theta}=U_{\theta} P U_{\theta}^{-1}=-h^{2} e^{-2 \theta} \Delta+V\left(e^{2 \theta} r^{2}\right)
$$


for $\theta$ in a complex neighborhood of 0 . We have:

$$
U_{\theta} P U_{\theta}^{-1}=\bigoplus_{\sigma=0}^{\infty} \bigoplus_{\tau=-\sigma}^{\sigma} U_{\theta} P(\sigma) U_{\theta}^{-1}=\bigoplus_{\sigma=0}^{\infty} \bigoplus_{\tau=-\sigma}^{\sigma} P_{\theta}(\sigma)
$$

with

$$
P_{\theta}(\sigma)=-h^{2} e^{-2 \theta}\left(\frac{\partial^{2}}{\partial r^{2}}+\frac{2}{r} \frac{\partial}{\partial r}-\frac{\sigma(\sigma+1)}{r^{2}}\right)+V\left(e^{2 \theta} r^{2}\right) .
$$

Simplicity of poles for the resolvent of the $P_{\theta}(\sigma)$ 's induces that for $P_{\theta}$. Let us consider the harmonic oscillator:

$$
Q=-h^{2} \Delta+|x|^{2}, \quad \mathscr{D}(Q)=H^{2}\left(\mathbb{R}^{3}\right) \cap \mathscr{D}\left(|x|^{2}\right) .
$$

We have $Q=\bigoplus_{\sigma=0}^{\infty} \bigoplus_{\tau=-\sigma}^{\sigma} Q(\sigma)$, where $Q(\sigma)$ is the self-adjoint extension from $\mathscr{C}_{0}^{\infty}\left(\mathbb{R}^{+}\right)$ of the operator $Q(\sigma)=-h^{2} \Delta(\sigma)+r^{2}$ with domain $\mathscr{D}(Q(\sigma))=H_{0, \sigma}^{1}\left(\mathbb{R}^{+}\right) \cap \mathscr{H}_{\sigma} \cap \mathscr{D}\left(r^{2}\right)$. The (simple) eigenvalues of $Q(\sigma)$ are given by $e_{k}(h)=h(2 \sigma-4 k+3)(k \geqq 0)$ and the corresponding eigenfunctions $e^{-r^{2} / 2 h} r^{\sigma} L_{k}^{\left(\sigma+\frac{1}{2}\right)}\left(r^{2} / h\right)$, where $L_{k}^{\left(\sigma+\frac{1}{2}\right)}$ is a Laguerre polynomial [NiOu, p. 48]. $k$ is called radial quantum number. The eigenstates of $Q$ are all degenerate (except the fundamental).

Theorem 3.1. Let $n=3$. Under hypothesis (1.1), (1.2)',(1.3), and (1.4), and for $\operatorname{Im} \theta>0$ small enough, then for all $C_{0}>0$ such that:

$$
-i e_{k}(h) \notin \partial D\left(0, C_{0} h\right) \forall k,
$$

and for all $h>0$ small enough, there exists a bijection $b=b(h)$ from

$$
\Gamma_{0}(h)=\left\{-i e_{k}(h) \mid k \in \mathbb{N}\right\} \cap D\left(0, C_{0} h\right)
$$

to the set of outgoing resonances of $P(\sigma)$ in $D\left(0, C_{0} h\right)$ such that

$$
b(E)-E=\mathcal{O}\left(h^{2}\right)(h \rightarrow 0) .
$$

In particular, the resonances of $P(\sigma)$ in $D\left(0, C_{0} h\right)$ are simple.

Idea of the Proof. The idea is to compare the resonances of $P(\sigma)$ to those of $\widetilde{P}(\sigma)=$ $-h^{2} \Delta(\sigma)-r^{2}$, as $h \rightarrow 0$ by mimicking the proof of $[\mathrm{BrCoDu}]$. As it goes along the same lines we leave it to the reader, except the following lemma (cf. Lemma 2.3 of $[\mathrm{BrCoDu}]$ ) which needs the assumption on the dimension (for $n \geqq 3$ it is equally true, but we don't know if it holds for $n=2$ and $\sigma=0$ ). We first perform another unitary transformation to put the operator $P(\sigma)$ in a normal form.

Let $U: L^{2}\left(\mathbb{R}^{+} ; r^{2} d r\right) \rightarrow L^{2}\left(\mathbb{R}^{+} ; d r\right)$ defined by $U(u)(r)=r u(r)$. We set

$$
\begin{aligned}
\Delta=U \circ \Delta(\sigma) \circ U^{-1}= & \frac{\partial^{2}}{\partial r^{2}}-\frac{\sigma(\sigma+1)}{r^{2}}, \quad P=U \circ P(\sigma) \circ U^{1} \text { and } \\
& P_{\theta}=U \circ P_{\theta}(\sigma) \circ U^{-1} .
\end{aligned}
$$

From now on, we work with $\Delta, P, P_{\theta}$. Let: $\Gamma_{\alpha}=\{\theta \in \mathbb{C}|\operatorname{Im} \theta|<\alpha\}$. We notice that $\left\{P_{\theta} \mid \theta \in \Gamma_{\alpha}\right\}$ is an analytic family of type $(A)$ with domain $\mathscr{D}(\Delta)$ for $\alpha>0$ small enough. The strategy of $[\mathrm{BrCoDu}]$ consists in decoupling the operator $P_{\theta}$ in an "interior" hamiltonian $P_{\theta}^{i}=-h^{2} e^{-2 \theta} \Delta-e^{2 \theta} r^{2}$ (quadratic approximation) on $L^{2}\left(\mathbb{R}^{+}\right)$and an "exterior" hamiltonian $P_{\theta}^{e}$ which is the Dirichlet realization of $P_{\theta}$ 
outside a small neighborhood of the origin in $\mathbb{R}^{+} . P_{\theta}^{i}$ has domain $\mathscr{D}\left(P_{\theta}^{i}\right)=\mathscr{D}(\Delta)$ $\cap \mathscr{D}\left(r^{2}\right)$ when $\operatorname{Im} \theta \neq 0$ and its properties give asymptotically, roughly speaking, those of $P_{\theta}$. We need the following:

Lemma 3.2. The family $\left\{P_{\theta}^{i} \mid \theta \in \Gamma_{\pi / 4} ; \operatorname{Im} \theta>0\right\}$ is an analytic family of type $(A)$ the spectrum of $P_{\theta}^{i}$ is independent of $\theta \in \Gamma_{\pi / 4}$ for $\operatorname{Im} \theta>0$ and given by $\operatorname{Sp}\left(P_{\theta}\right)=-i \operatorname{Sp}\left(-h^{2} \Delta+r^{2}\right)$.

Proof. There suffices to check that $\left.\left\{\left(P_{\theta}^{i} \mid \theta=i \beta, \beta \in\right] 0, \pi / 4\right]\right\}$ is a family of closed operators on $L^{2}$, with domain $\mathscr{D}(\Delta) \cap \mathscr{D}\left(r^{2}\right)$. For $\beta=\pi / 4$, this is obvious. As $\Delta$ and $r^{2}$ are self-adjoint, and thus closed, there suffices to show in turn that if $0<|\beta|<\frac{\pi}{4}$,
then

$$
\left\|P_{\theta}^{i} u\right\|^{2}+\|u\|^{2} \geqq C_{\theta}\left(h^{4}\|\Delta u\|^{2}+\left\|r^{2} u\right\|^{2}\right) \quad\left(C_{\theta}>0\right),
$$

for $u \in \mathscr{D}(\Delta) \cap \mathscr{D}\left(r^{2}\right)$. But in operator sense

$$
\begin{aligned}
\left|P_{\theta}^{i}\right|^{2}= & h^{4} \Delta^{2}+r^{4}+h^{2} \cos 4 \beta\left(r^{2} \Delta+\Delta r^{2}\right)-2 i h^{2} \sin 4 \beta\left(r \partial_{r}+\partial_{r} r\right) \\
& \geqq\left(h^{4} \Delta^{2}+r^{4}\right)(1-|\cos 4 \beta|)-2 i h^{2} \sin 4 \beta\left(r \partial_{r}+\partial_{r} r\right) .
\end{aligned}
$$

On the other hand

$$
\begin{aligned}
\mp 2 i h^{2} \sin 4 \beta\left(r \partial_{r}+\partial_{r} r\right) & \geqq-2 h|\sin 4 \beta|\left(r^{2}-h^{2} \Delta\right)+2 h^{3}|\sin 4 \beta| \frac{\sigma(\sigma+1)}{\mathrm{r}^{2}} \\
& \geqq-2 h|\sin 4 \beta|\left(r^{2}-h^{2} \Delta\right)
\end{aligned}
$$

(here we use the assumption on the dimension, which rules out the existence of a repulsive term). Inequality (3.3) easily follows as in [ $\mathrm{BrCoDu}]$. It also shows that $P_{\theta}^{i}$ is an operator with compact resolvent for $0<|\beta|<\pi / 4$ (cf. the proof Theorem XII-16 in [ReSi]). There follows from the analyticity of $P_{\theta}^{i}$ that its spectrum is independent of $\theta$, which proves the lemma.

There follows in particular from Lemma 3.2 that the spectrum of $P_{\theta}^{i}$, for $\operatorname{Im} \theta>0$ consists in the simple eigenvalues

$$
\lambda_{\theta}(h)=-i h(2 \sigma+4 k+3) \quad(k \in \mathbb{N}) .
$$

The proof then again goes as in $[\mathrm{BrCoDu}]$.

We conjecture that the simplicity of poles for a radial symmetric potential holds for all $h$. To show degeneracies, it is then necessary to break the radial symmetry of the potential, which justifies the general procedure of perturbation set up in Sect. 1. We just showed that assumption (1.24) is always verified. We have the following result, similar to this of Sect. 2:

Theorem 3.3. For $n=3$, let $P$ satisfy hypotheses (1.1), (1.2)', (1.3), and (1.4) and let $\lambda(h)$ be a resonance of $P$ close to $\lambda_{0}(h)=-i h(2 \sigma+3)$ in the sense of $(1.7)$, with (geometric) multiplicity $q=2 \sigma+1\left(\sigma \in \mathbb{N}^{*}\right)$ satisfying also hypothesis (1.9). Then there exists a compact perturbation $\Delta V(x, h)$ of $P$ such that the meromorphic extension (in the sense of analytic dilations) of the resolvent $(\widetilde{P}-z)^{-1}$ of $\widetilde{P}=P$ $+\Delta V(x, h)$ close to $z=0$ in the half plane $\operatorname{Im} z<0$ has a pole of order $q$, for any $h>0$ small enough, in a neighborhood of the semi-simple resonance $\lambda(h)$.

We proceed now to sketch a proof of Theorem 3.3. The resonant functions of $P(\sigma)$ associated to the outgoing resonance $\lambda_{0}(h)=-i h(2 \sigma+3)$ are given by

$$
f_{\beta}(x)=C_{\sigma} h^{-(2 \sigma+3) / 4} e^{i r^{2} / 2 h} r^{\sigma} Y_{\beta}(\omega), \quad \beta=(\sigma, \tau), \quad x=r \omega .
$$


Let $\Psi_{\beta}(x)$ be the resonant functions of $P(\sigma) \otimes \operatorname{Id} \mathscr{Y}_{\sigma}$ associated to resonances of $P(\sigma)$ close to $\lambda_{0}(h)$ in the sense of $(1.7)$. We can easily prove, with similar arguments to those giving Lemma 1.2 and Proposition 1.4:

Lemma 3.3. With notations of Proposition 1.3 we have:

$$
\left(\Delta V \Psi_{\beta} \mid \Psi_{\gamma}^{*}\right)=\left(\Delta V f_{\beta} \mid f_{\gamma}^{*}\right)+\mathcal{O}\left(h^{l-m-1}\right), \quad \beta=(\sigma, \tau), \quad \gamma=\left(\sigma, \tau^{\prime}\right)
$$

With the same notations, and under hypothesis (1.1) to (1.4) and (1.9) we have

$$
\begin{aligned}
\left(\widetilde{P}_{\theta} v_{\beta} \mid v_{\gamma}^{*}\right)= & \lambda(h) \delta_{\beta \gamma}+h^{v}\left(\sum_{m} i^{m} \frac{\Gamma(\sigma+m+3 / 2)}{\Gamma(\sigma+3 / 2)}\right. \\
& \left.\times \int_{S^{2}} q_{2 m}(\omega, 0) Y_{\beta}(\omega) Y_{\gamma}(\omega) d \omega+\mathcal{O}(h)\right), \quad \beta=(\sigma, \tau), \gamma\left(\sigma, \tau^{\prime}\right) .
\end{aligned}
$$

We then proceed to an analysis totally analogous to this of Sect.1.2. The first step consists to show the independence of products $\left(Y_{\beta}(\omega) Y_{\gamma}(\omega)\right)_{\beta, \gamma}$.

Lemma 3.4. For all $\sigma \in \mathbb{N}$ the $\left(Y_{\beta}(\omega) Y_{\gamma}(\omega)\right)_{\beta, \gamma}(-\sigma \leqq \beta, \gamma \leqq \sigma)$ form a set of $q(q+1) / 2$ linearly independent trigonometric polynomials. Here $q=2 \sigma+1$.

The proof of this lemma ultimately uses known properties of Legendre polynomials (cf. [NiOu]) and elementary trigonometric functions, it is somewhat lengthy but straightforward and so we leave it to the reader.

The next step, as in Sect. 2, consists in noticing that the products $\left(Y_{\beta}(\omega) Y_{\gamma}(\omega)\right)_{\beta, \gamma}$ are even functions on $S^{2}$ [either by a direct computation, either by noticing that $e^{-r^{2}} r^{2 \sigma} Y_{\beta}(\omega) Y_{\gamma}(\omega)$ is a linear combination of the $\left(\Phi_{\alpha}(x) \Phi_{\alpha^{\prime}}(x)\right)_{|\alpha|=\left|\alpha^{\prime}\right|=\sigma}$, then in establishing a density result of trigonometric polynomials $\mathscr{D}_{s}(s=1.2)$ in $L_{+}^{2}\left(S^{2}\right)$, e.g. by an argument of approximation in the topology of uniform convergence for even functions in $\mathscr{C}_{0}^{\infty}\left(S^{2}\right)$ whose support avoids the equator $\theta=\pi / 2$ and a meridian, by polynomials of $\mathscr{D}_{s}$ (Stone-Weierstrass). Again the proof is left to the reader as an exercise. We then achieve the proof as in Sect. 2 by a transversality argument.

\section{Appendix}

Here we show that the hypothesis (1.9) of separation in the spectrum is generally fulfilled, under a condition relative to $V^{(4)}(0)$. We actually show a more precise result concerning the $E_{n, l}$ levels classification of resonant states for a radial potential, as $h \rightarrow 0$, which seemed to us to be new, even in the self adjoint case (see however [DaPa]).

Proposition A1. Let $P=-h^{2} \Delta+V(x)$ on $\mathbb{R}^{n}$ with odd $n \geqq 3$ satisfying hypotheses (1.1), (1.2)', (1.3), and (1.4), and $V^{(4)}(0) \neq 0$.

(i) If $\lambda_{0}(h)=-i h(2 \sigma+n)$ is a resonance of the hamiltonian $P_{0}=-h^{2} \Delta-|x|^{2}$ associated to $P$, then there exists $q_{0}=\left(\begin{array}{c}\sigma+n-1 \\ \sigma\end{array}\right)$ resonances of $P$ close to $\lambda_{0}(h)$ in the sense of (1.7) counted with their multiplicity

$$
d_{k}=\left(\begin{array}{c}
\sigma^{\prime}-n-1 \\
\sigma^{\prime}
\end{array}\right)-\left(\begin{array}{c}
\sigma^{\prime}-n-3 \\
\sigma^{\prime}-2
\end{array}\right)
$$

where $\sigma^{\prime}=\sigma-2 k$ for $k=0, \ldots,[\sigma / 2]$ and such that $\lambda_{m, k}(h)=-i h m+h^{2} \alpha_{m, k}+\mathcal{O}\left(h^{3}\right)$ $\left(\alpha_{m, k} \in \mathbb{R}\right)$ with $m=2 \sigma+n$ and $\alpha_{m, k} \neq \alpha_{m, k^{\prime}}$ for $k \neq k^{\prime}$. 
(ii) In particular, if $n=3$, with the notations of Sect. 3 , the $k^{\text {th }}$ resonance of $P(\sigma)=-h^{2} \Delta(\sigma)+V\left(r^{2}\right)$ is of the form $\lambda_{2 \sigma+4 k+3, k}(h)$ with $k \in \mathbb{N}$.

Proof. We use analytic dilations. We compute, in a somewhat formal way, the asymptotic expansions for the resonances of $P$ at order 2 in $h$ (their existence being justified in [Sj]). For $\operatorname{Im} \theta>0$ we first perform a Bargmann transform:

$$
T_{\theta} u(x)=\int_{\mathbb{R}^{n}} e^{i e^{2 \theta}\left(x^{2}+y^{2}+2 \sqrt{2} x y\right) / 2 h} u(y) d y
$$

for $u \in L^{2}\left(\mathbb{R}^{n}\right), x \in \mathbb{C}^{n}$. Then it is easy to see that if we set $W\left(|y|^{2}\right)=V\left(|y|^{2}\right)+y^{2}$, the conjugate operator $Q_{\theta}=T_{\theta} P_{\theta} T_{\theta}^{-1}$ is the formal pseudo-differential operator defined by:

$$
Q_{\theta}=-i h\left(2 x \partial_{x}+n\right)+\mathrm{Op}\left(W\left(e^{2 \theta}\left(e^{-2 \theta} h \xi-x\right)^{2} / 2\right)\right),
$$

where $x \partial_{x}=r \partial_{r}$ denotes the radial holomorphic vector field $x_{1} \partial_{x_{1}}+\ldots+x_{n} \partial_{x_{n}}$. We still denote by $Q_{\theta}$ the image of $Q_{\theta}$ by the transform $x \rightarrow h^{-1 / 2} x$ and $\mu=W^{\prime \prime}(0) / 8$, where $W$ is considered as a function of $t=r^{2}$; in the sense of pseudo differential operators we have:

$$
\left.Q_{\theta}=-i h\left(2 r \partial_{r}+n\right)+\mu h^{2} \mathrm{Op}\left(\left(e^{-\theta} \xi-\theta^{\theta} x\right)\right)^{4}\right)+\mathcal{O}\left(h^{3}\right) .
$$

At the level of principal symbol we simply get:

$$
Q_{\theta}=-i h\left(2 r \partial_{r}+n\right)+\mu h^{2}\left(-e^{-2 \theta} \Delta+2 i r \partial_{r}+i n+e^{2 \theta} r^{2}\right)^{2}+\mathcal{O}\left(h^{3}\right) .
$$

We look for eigenfunctions of $Q_{\theta}$ as classical symbols in $h$, valued in polynomials in $x$. At the first order in $h$ the eigenfunctions of $Q=-i h\left(2 r \partial_{r}+n\right)$ are the homogeneous polynomials $q_{\sigma}(x)$ of degree $\sigma \in \mathbb{N}$. Following [V,p.446], we have the (unique) decomposition:

$$
q_{\sigma}(x)=\sum_{k=0}^{[\sigma / 2]} r^{2 k} h_{\sigma-2 k}(x),
$$

where $h_{\sigma-2 k}$ is an homogeneous harmonic polynomial of degree $\sigma-2 k$. Then:

with

$$
\begin{aligned}
Q_{\theta}\left(q_{\sigma}\right)(x)= & -i h(2 \sigma+n) q_{\sigma}(x) \\
& +\mu h^{2} \sum_{k=0}^{[\sigma / 2]}\left(a_{k}\left(r^{2}\right) r^{2 k}+b_{k} e^{-2 \theta} r^{2 k-2}+c_{k} e^{-4 \theta} r^{2 k-4}\right) h_{\sigma-2 k}(x)+\mathcal{O}\left(h^{3}\right)
\end{aligned}
$$

$$
\begin{gathered}
a_{k}\left(r^{2}\right)=\left(e^{2 \theta} r^{2}+i m\right)^{2}+4 i e^{2 \theta} r^{2}-4 k(m-2 k-2)-2 m, \\
b_{k}=-4 i k(m-2 k-2)(m-2), \\
c_{k}=4 k(k-1)(m-2 k-2)(m-2 k-4),
\end{gathered}
$$

where we have set: $m=2 \sigma+n$. We then look for the eigenfunctions of $Q_{\theta}$ in the form

$$
u_{k}(x)=r^{2 k} h_{\sigma-2 k}(x)\left(1+h f_{k}\left(r^{2}\right)+\mathcal{O}\left(h^{2}\right)\right)
$$

and the eigenvalues:

$$
\lambda_{k}(h)=-i h m+h^{2} \alpha_{m, k}+\mathcal{O}\left(h^{3}\right)
$$

for $k=0,1, \ldots,[\sigma / 2]$. Substituting (A3) and (A4) in (A1) we get to the second order in $h$, after simplification and setting as before: $t=r^{2}$ :

$$
-4 i t f_{k}^{\prime}(t)+\mu\left(a_{k}(t)+b_{k} t^{-1} e^{-2 \theta}+c_{k} t^{-2} e^{-4 \theta}\right)=\alpha_{m, k} .
$$


If we write $f_{k}(t)=\sum_{j=-k}^{\infty} \beta_{j} t^{j}$ the latter equation gives by identificating the coefficients of the powers of $t$ :

$$
f_{k}(t)=i \mu c_{k} / 8 e^{-4 \theta} t^{-2}+i \mu b_{k} / 4 e^{-2 \theta} t^{-1}+\mu(m+2) / 2 e^{2 \theta} t-i \mu / 8 e^{4 \theta} t^{2}+\text { const }
$$

if the following compatibility condition is satisfied:

$$
\mu\left(m^{2}+2 m+4 k(m-2 k-2)\right)+\alpha_{m, k}=0 .
$$

This relation determines the second term of the asymptotic expansion of $\lambda_{k}(h)$. Further, if there exists $k$ and $k^{\prime}$ such that $\alpha_{m, k}=\alpha_{m, k^{\prime}}$, we get:

$$
\left(k-k^{\prime}\right)\left(m-2\left(k+k^{\prime}\right)-2\right)=0,
$$

whence $k=k^{\prime}$ for $m=2 \sigma-n$ is odd if $n$ is. Then we proved point (i). Point (ii) easily follows by inverse Bargmann transform and polar coordinates.

\section{References}

[AgCo] Aguilar, J., Combes, J.M.: A class of analytic perturbations for one-body Schrödinger hamiltonians. Commun. Math. Phys. 22, 269-279 (1971)

[BaCo] Balsev, E., Combes, J.M.: Spectral properties of many-body Schrödinger operators with dilation analytic interactions. Commun. Math. Phys. 22, 280-294 (1971)

[BrCoDu] Briet, P., Combes, J.M., Duclos, P.: On the location of resonances for Schrödinger operators in the semi-classical limit. II. Commun. Part. Diff. Eq. 12, 201-222 (1987)

[DaPa ] Daubechies, I., Paul, T.: Wavelets and applications. Proceeding VIII-th Int. Congress on Math. Phys. Mebkout, M., Seneor, R. (eds.) pp.675-680, Singapore: World Scientific 1987

[G] Gantmacher, M.: Theory of matrices. II. Chelsea 1972

[HeSj] Helffer, B., Sjöstrand, J.: Resonances en limite semi-classique. Memoire de la S.M.F., (1986), Tome 114(3)

[HeMa] Helffer, B., Martinez, A.: Comparaison entre les diverses notions de résonances. Helvetica Phys. Acta 60, 992-1003 (1987)

[KaRo] Kaidi, N., Rouleux, M.: Resonances multiples en limite semi-classique. Proceedings: Journées des Equations aux dérivées partielles. St. Jean de Monts (1988). Société Math. de France

[M] Mackey, G.M.: Quantum mechanics from the point of view of the theory of group representations. Lect. Appl. Math. 21, 219-253 (1985)

[NiOu] Nikivorov, A., Ouvarov, V.: Théorie élémentaire des fonctions spéciales. Editions Mir (Moscou) 1974

[Ra] Ramm, A.G.: Perturbations of resonances. J. Math. Anal. Appl. 88, 1-7 (1982)

[ReSi] Reed, M., Simon, B.: Methods of modern mathematical physics. IV. Analysis of operators. New York: Academic Press 1978

[Ro] Rouleux, M.: Diffraction analytique sur une variété à singularité conique. Commun. Part. Diff. Eq. 11, 947-988 (1986)

[Sj] Sjöstrand, J.: Semi-classical resonances generated by non-degenerate critical points. Lect. Notes in Mathematics, vol. 1256, pp. 402-429. Berlin, Heidelberg, New York: Springer 1987

[V] Vilenkin, N.J.: Special functions and the theory of group representation. Trans. Math. Mono. Vol. 22. Providence, R.I.: Am. Math. Soc. 1968 